\title{
A Selective Bilingual Advantage in High School Students
}

\author{
Laura Khotemlyansky ${ }^{1}$ and Jill Berge ${ }^{1 \#}$ \\ ${ }^{1}$ Lake Washington High School, Kirkland, WA, USA \\ "Advisor
}

$\underline{\text { ABSTRACT }}$

The present study investigates the impacts of bilingualism on academic performance among high school students by using cognitive assessment scores as proxies for GPAs. As seminal works, finding a constant bilingual advantage (BA), are being increasingly disproved in favor a selective BA, task demand in working memory (WM) was introduced to examine this. A mixed method was used. Participants completed a self-assessment survey, rating their academic performance and beliefs regarding the bilingual advantage hypothesis. Then, the flanker and modified flanker tasks tested participants' WM. Using measurements of the means, medians and p-test, it was discovered that bilingual students only outperformed monolinguals on the low demand regular flanker task while equally on all other tasks, not in accordance with the Executive Control Theory (ECT) commonly supported in recent works. Additionally, the survey revealed that both monolingual and bilingual students recognize the positive impacts of language on cognition, but not the application of cognition to increased school performance. By bringing awareness to this relationship, this could encourage more students to study a foreign language in high school, bridging the cognitive-linguistic gap.

\section{Introduction}

The US is often dubbed a "nation of immigrants", and rightfully so. The 47 million immigrants living in America constitute the largest immigrant population within a single country: 19\% (Waters \& Gerstein, 2015). In 2019, the United Nations Migration Report found that as the rate of immigration to the US increased, so did the population that spoke a language other than English at home. (International migration report, 2019). According to the U.S. Census Bureau, $20 \%$ of Americans are bilingual, doubling in the last forty years (America speaks, 2000). More specifically, bilingual students represent $22 \%$ of the population in American schools, highlighting the importance of addressing the changing classroom demographic (The number of bilingual kids, 2018). Thus, the education of bilinguals must be investigated. To best understand such education, the differences in cognition-the process of knowledge acquisition — must be identified between bilinguals and monolinguals as these mental processes characterize learning (Adesope et al., 2010).

\section{Literature Review}

\section{Inconsistencies in Research}

Despite numerous studies, there is little agreement in the field regarding the impacts of bilingualism on executive function (EF), a subset of cognitive skills involved in learning.

Seminal studies have largely established a constant bilingual advantage (BA), suggesting that the language practices of bilinguals-suppression or inhibition of the dormant language and shifting in a multi-lingual environment—enhance their cognition, causing bilinguals to outperform monolinguals on EF tasks, including schoolwork. 
However, such results are no longer reproducing in recent studies as 80\% of findings on the BA after 2011 have discovered null results (Paap et al., 2015). These inconsistencies were examined by Paap et al. (2015) in a metaanalysis of previous studies to determine questionable research practices (QRPs). Common QRPs include confirmation bias through continued data collection to avoid null results, unrepresentative samples, and small population sizes. Research with larger sample sizes, contrasting the small sample population common in seminal works, observes less or no BA, while the latter produces significantly greater amounts of positive results. As sample size increases, the null model will always be rejected if it is false. Thus, results from large populations indicate no possibility of a BA from experimental errors. Since seminal works do not repeat findings through direct replication when proper baselines, sample sizes, and controls are used, this puts into question the discovered BA.

Another meta-analysis conducted by Adescope et al. (2010) narrowed down a cohort of 5,185 articles based on an initial Boolean search to a final 63, aided by selection criteria to ensure methodologically sound studies, eliminating QRPs. A cognitive advantage amongst bilinguals was observed in all subcategories of attention control, working memory (WM), metalinguistic/metacognitive awareness, problem solving, abstract/symbolic reasoning, and creative/divergent thinking. All categories except WM displayed heterogeneous results, demonstrating higher variability in effect size than expected from sampling error, prompting moderator analysis. WM was excluded from further analysis due to the markedly different dependent variables and small number of studies included in the meta-analysis. This demonstrates both the unique characteristics of WM in comparison to other cognitive correlates and the lack of methodologically comprehensive WM studies, prompting further investigation of this specific subset of EF. As unbalanced bilinguals were excluded from the selection criteria, further inquiry is required for bilinguals with diverse proficiency.

\section{Task Demand and Working Memory}

Following Paap et al.'s (2015) methodological framework, Antón et al. (2019) conducted a study testing the reliability of the BA in Basque and Spanish young adults. Bilinguals were matched on factors including socio-economic status (SES), proficiency levels, ethnicity, IQ levels, immigrant status, etc., eliminating uncontrolled variables. Testing bilinguals and monolinguals on standard tasks in bilingual research, EF and WM were tested. Namely the flanker, Simon, Verbal Stroop and Numerical Stroop tasks tested EF while the forward and backward Corsi and digit span tests measured WM. Extensive correlation and bootstrapping analysis revealed a null model for BA, reflecting a general trend of growing controversy on the previously recognized postulate. This suggests that the BA observed in previous studies was derived from external factors, controlled by Antón et al. However, a BA was observed in backward WM tasks, attributed to the increased difficulty of the task, called task demand.

Therefore, the selective BA in WM should be further examined.

As necessitated by Antón et al.'s study, Lu et al. (2019) tested the influence of task demand on WM, analyzing the two hypotheses arising from increased cognitive load. The first predicts a disadvantage for bilinguals due to the overload of their WM function, while the other expects an advantage as the overload strengths the efficiency of bilinguals' storage mechanism. The second hypothesis aligns with the executive control theory (ECT), stating that when executive resources are sufficiently challenged, they are distributed more efficiently, improving cognitive performance. The study tested two types of inhibitions, a sub-system of EF, co- dependent and co-occurring with WM. The tasks used required participants to simultaneously analyze the information to perform proper inhibition, while storing the parameters for certain actions, thus incorporating WM. The study included two experiments, each testing a different inhibition with low and high demand tasks. In both experiments, a BA was only observed in the highest demand WM tasks. Furthermore, in experiment two, the BA only occurred in the highest demand trial of the Conditional-GNG task, not the task as a whole. Bilingual and monolingual performance was equal in low demand tasks. Lu et al.'s findings explain the irregularities observed in previous studies of the BA, presenting a new nuance of BA that must be further examined. Additionally, these findings support the ECT and second hypothesis of cognitive load. Lu et al. shows that the BA must be activated 
to improve cognitive control, explaining Antón et al.'s findings of BA only in backward WM tasks: the only tasks that sufficiently challenged cognition.

Gutiérrez-Clellen et al. (2004) came to a contradictory conclusion than Antón et al. and Lu et al., comparing the performance of 44 Latino children on verbal WM tasks. Language groups of varying proficiency were tested on two processing tasks, both revealing a null best fit model, refuting the ETC and BA. Correlation analysis showed similar cognitive-linguistic operations involved in both tasks. Gutiérrez-Clellen et al. acknowledge that the tasks may not have challenged EF enough to produce a BA, encouraging further research within a heterogeneous bilingual population and with increased cognitive demands.

\section{Cognition and Education}

While research around the BA in EF is largely disputed, findings regarding the correlation of cognition and education are in agreement.

A population-based study conducted by Guhn et al. (2016) investigated the school readiness profiles of kindergarteners from three bilingual groups in comparison to two monolingual groups. Children were categorized based on their ELL status. Much like in Antón et al.'s study, the research controlled for SES and the nested classroom structure. The data was obtained from population-level databases, using teachers' ratings of students on the Early Development Instrument (EDI). The EDI comprises predictors of school success based on cognitive and social domains. Students were tested on the EDI domains and subdomains in the language of instruction. Multilevel hierarchical linear modeling of the data revealed that bilinguals scored significantly higher on 8 out of the 15 EDI subdomains. Namely prosocial behavior, respect and responsibility, social competence, independence and adjustment, communication, and all cognitive subdomains. This supports the BA as the most significant and consistent high ratings of bilinguals were observed in cognition, opposing findings of a constant BA. The rest of the domains showed equal performance for bilinguals and monolinguals. Future school readiness research among older students is necessary to track the impact of bilingualism on an older age group in a non-developmental setting. Additionally, it is important to consider that teacher ratings were used as proxy for predictors of academic success, necessitating other measurements of students' school performance.

Employing the nationally representative Early Childhood Longitudinal Study Data (ECLS-K) database, Han (2012) analyzed the role of bilingualism on academic development trajectories in early school years. Bilinguals were divided into language and proficiency groups with ELL and immigrant status used as proxies for categorization. Han observed the reading and math scores within each language group from tests administered twice per school year. To restrict analysis to specific language groups, only data on children with Latino or Asian backgrounds and monolingual native-born non-Hispanic whites (the reference group) were included, limiting the sample to 16,380 students. Overall, the reference group scored the highest on initial reading and math tests. Model 3 $(\mathrm{p}<0.001)$ provided the best fit for the data and included the most controls, demonstrating that while the reference group initially had higher scores, bilinguals had a significantly higher rate of change in their academic trajectories, closing the knowledge gap. These findings provide conflicting stances on the BA as bilingual students had lower or similar initial test scores to English-monolinguals, while improving at a faster rate.

\section{Further Research}

These studies demonstrate the inconsistencies of BA findings that must be addressed to examine bilingual education. In particular, the impact of bilingualism on task dependent WM must be examined to study the presence of a selective BA, more prevalent in recent studies than seminal ones. While many studies use population-level databases like the ECLS-K for bilingual research, they do not represent students past fifth grade. Therefore, it is necessary to track the BA in high school students, the least studied age group for bilingual research, on an instantaneous rather than developmental basis. While studies extract information from the participants to place proper controls, 
little research has tested the perceptions of participants on bilingualism or the BA and their impacts on school performance. Additionally, no studies have cross-referenced cognitive assessment scores to students' perceptions of their academic abilities to determine their accuracy. This gives rise to the question, "How does bilingualism impact academic performance in comparison to monolingualism?" This can be investigated in three parts, namely the perception of high school students of the BA, their self-assessment of academic performance in relation to cognitive scores, and the impact of task demand on WM performance.

\section{Methods}

To examine the impact of bilingualism on academic performance, the researcher employed a mixed-method, multi-step approach in which participants completed a survey and subsequent cognitive assessments.

All students in Lake Washington High School were sent an initial email through the school administrator containing information regarding the study and a link to the Microsoft Forms survey with an included consent form. Survey questions were branched based on participants' self-classification of bilingual or monolingual. The surveys for the two language groups were similar, however, monolinguals were also questioned whether they would consider learning another language. The survey included three sections: bilingual or monolingual classification, self-assessments, and steps for further participation, provided in Appendix A. Within the self-assessment section, students evaluated their academic abilities and BA beliefs. No controls based on SES or gender were collected, aligning with previous research.

Namely, Bialystok and Calvo (2014), who found no relationship between bilingualism and SES, concluding that the overriding positive influences of bilingualism on EF were not masked by poverty. Additionally, Adescope et al.'s (2010) findings corroborate as the meta-analysis of cognitive studies revealed that mean effect sizes did not differ statistically with varying SES while Engel de Abreu et al. (2012) found a persistent BA even when controlling for cultural factors. Finally, Guhn et al.'s (2016) findings show a null effect of gender on cognition, demonstrating an independent relation of cognition to control factors.

To collect self-evaluations, students ranked their performance from well below average, below average, average, above average and well above average on their overall academic performance, overall curriculum comprehension and material memorization. Thus, self- assessment was suitable for this data collection as scores were not assumed to be precise measurements of student performance. Instead, cognitive scores and self-assessments were cross- referenced to determine the accuracy of the self-evaluations, satisfying the aims of the second subresearch question. Additionally, Guhn et al. (2016) demonstrated that evaluation-based categorization of student performance is reliable for determining a BA. The study used teachers' ratings of students on the EDI instrument to predict school readiness. The correlative analysis revealed the same statistical similarity as the teacher evaluations, showing validity for this method of data collection.

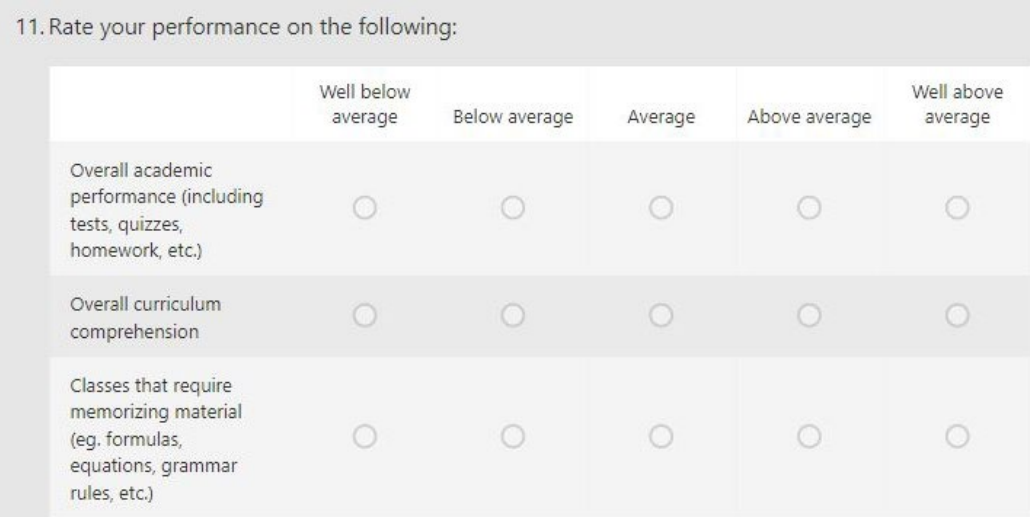

Figure 1. Self-evaluation criteria used in comparison to cognitive score 
Then, participants were questioned on their beliefs of the BA to investigate public perception, aligning with the aim of the first sub-question. To satisfy these aims, questions regarding the performance of bilinguals compared to monolinguals in school, the impacts of bilingualism on cognition, and the willingness of monolinguals to learn another language to obtain the cognitive advantages associated with bilingualism (give only to monolinguals through branching) were used. Questions were based on the definitions of bilingualism and the BA as explained by Paap et al. (2015), corroborated by the postulate used for BA in previous research.

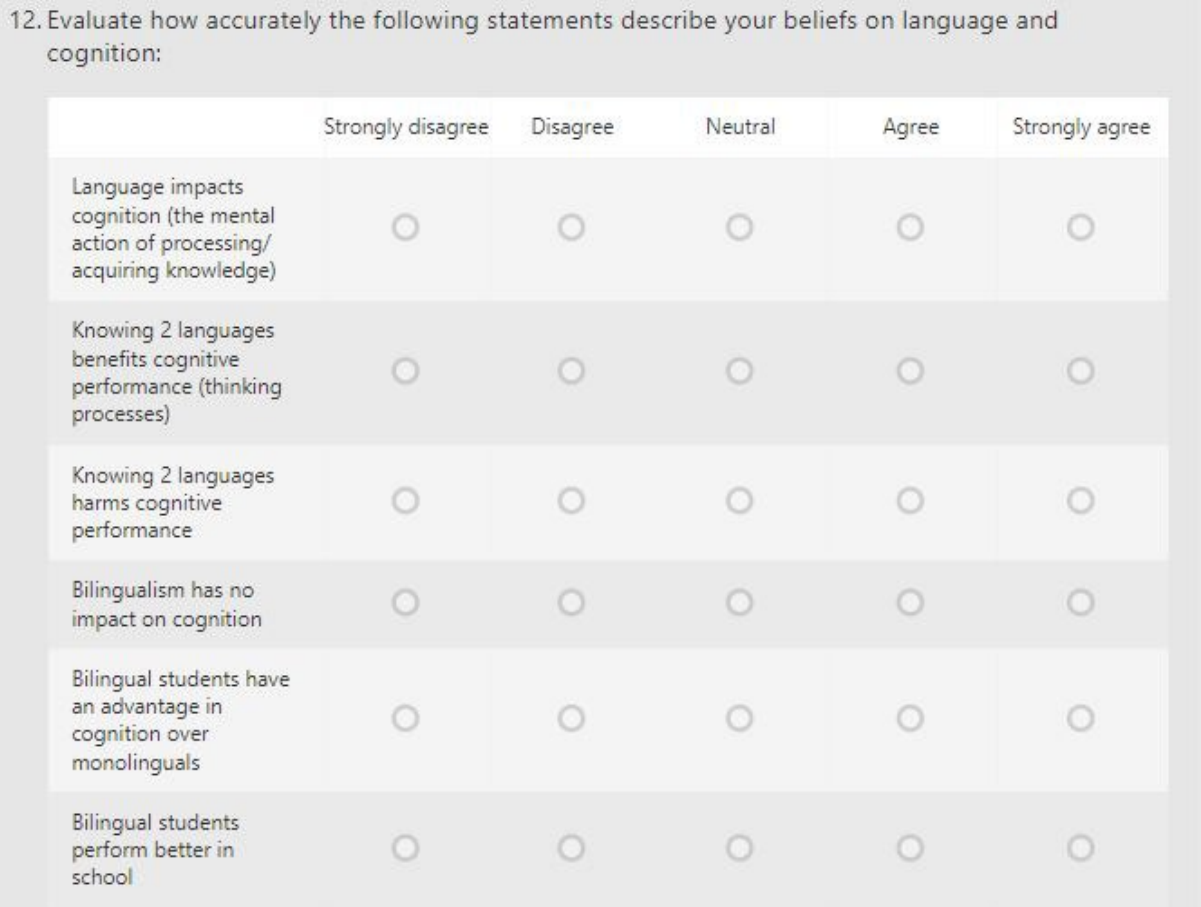

Figure 2. Questions regarding the bilingual advantage and its impacts on education

If participants expressed interest in further participation in the cognitive tests at the end of the survey, they received an email from the investigator with additional information in addition to a consent form. After the investigator received the signed consent form, participants were sent the link to the flanker and modified flanker tasks.

Cognitive WM assessment scores were used as proxies for academic performance instead of GPAs as such information could not be collected due to privacy constraints. While the existence of a BA is disputed, the direct relationship between increased cognitive performance, regardless if it is caused by language factors, and resulting improved school trajectories, is an accepted postulate. Guhn et al. (2016) supports this conclusion by investigating the connection between bilingualism and school readiness profiles based on the EDI instrument, compromised of school success predictors. Cognitive subdomains were used to predict better school performance, independent of language, showing the positive impacts of cognition on education, supported by multilevel hierarchical linear modeling. Additionally, a comprehensive study by Hair et al. (2006) used the ECLS-K database to test which kindergarten profiles predicted greater school readiness. In Hair's study, students were characterized into various development groups based on five rating domains. Children scoring lower on language and cognition were labeled as health risks, emphasizing their corresponding nature, justifying proxy categorization.

Employing a common cognitive task used in bilingual research, like Antón et al. (2019), the Eriksen flanker task was used to assess WM. A distinctive addition to the flanker task was the introduction of a task demand condition, increasing WM load and investigating the third aim of the research question. Like Lu et al. (2019), the flanker task tested low demand while the modified flanker task was used for high demand. Contrastingly, Lu et al. 
employed additional cognitive tasks to test specific aspects of WM: interference suppression and response inhibition. However, in this study, WM was investigated as a whole, rather than its components. Therefore, like Antón et al., who independently investigated EF and WM, only the flanker task was used and the corresponding higher demand task.

The flanker task was structured based on the original study conducted by Eriksen \& Eriksen (1974). This study tested visual information processing by observing the impact of flanker letters on participants' responses to the central stimuli, outlining the cognitiveassessment that was later named the Eriksen flanker task. It utilized a row of letters in which the central letter was the only response stimuli. The target was flanked on either side by two noise letters with certain conditions including congruent and incongruent trials. Right or left levers were pressed depending on the central letter S, C, H or K (Eriksen \& Eriksen 1974). However, to ensure accessibility to all language groups, letters were substituted for arrows.

The flanker and modified flanker tasks, provided in Appendix B, were created using html and JavaScript in Microsoft Visual Studio. Reaction times and accuracy scores were saved by the server tied to the database using APIs. The data was converted into an Excel file for further analysis. Prior to each cognitive test, participants read instructions for the task and completed 15 demo trials. Practice trials were kept to a minimum in accordance with Lu et al.'s findings, revealing the most prominent BA occurred when responses were not automatic. Instead, cognitive resources had to be under competition, as in the ECT. Therefore, practice trials ensured participants understood the directions, but did not develop an automatic response. Then, participants completed 75 trials of each assessment.

In the flanker task, participants had to press an arrow on the keyboard corresponding to the direction of the central arrow, ignoring the flankers. In the modified flanker task, to increase task demand, the space bar had to be pressed if at least one $\mathrm{X}$ was present among the flankers. The amount of Xs present per 75 trials was randomized to prevent predictable, and thus automated responses. WM was used to memorize the conditions under which to press certain keys. Furthermore, WM demand was increased in the modified flanker when the no-go condition was introduced as participants had to additionally remember to press the space bar for a X.

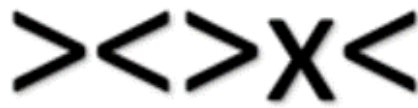

Correct Response:

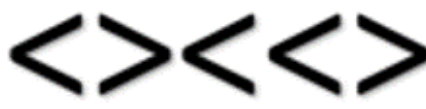

Correct Response:

\section{space bar}

Figure 3. Responses necessitated by the modified (left) and regular (right) flanker

Lake Washington High School was selected for the sample population to examine a nationally representative student sample. The national average bilingual student population is $22 \%$, corresponding to that of Washington state (Children Who Speak A Language, 2020). Furthermore, King County is a nationally representative county with a $19.1 \%$ bilingual student population and a diverse sample consisting of 170 languages (Felt 2017).

\section{Results}

Data was analyzed in three categories in relation to the sub-research questions. All findings were compared among language groups to investigate the impacts of bilingualism. 


\section{Student Perception}

52 bilinguals and 48 monolinguals completed the self-assessment survey, creating a homogeneous cohort. Thus, this sample size accurately represents perceptions of both language groups. The criteria for a statement to be generally accepted among a language group required the majority $(>50 \%)$ of responses to classify their beliefs on the statement as either strongly agree or agree. Similarly, for a statement to be refuted, the majority $(>50 \%)$ of responses had to indicate disagreement or strong disagreement. Based on this criterion, both bilinguals $(82.40 \%)$ and monolinguals $(81.20 \%)$ demonstrated agreement that language impacts cognition, and more specifically, benefits it.

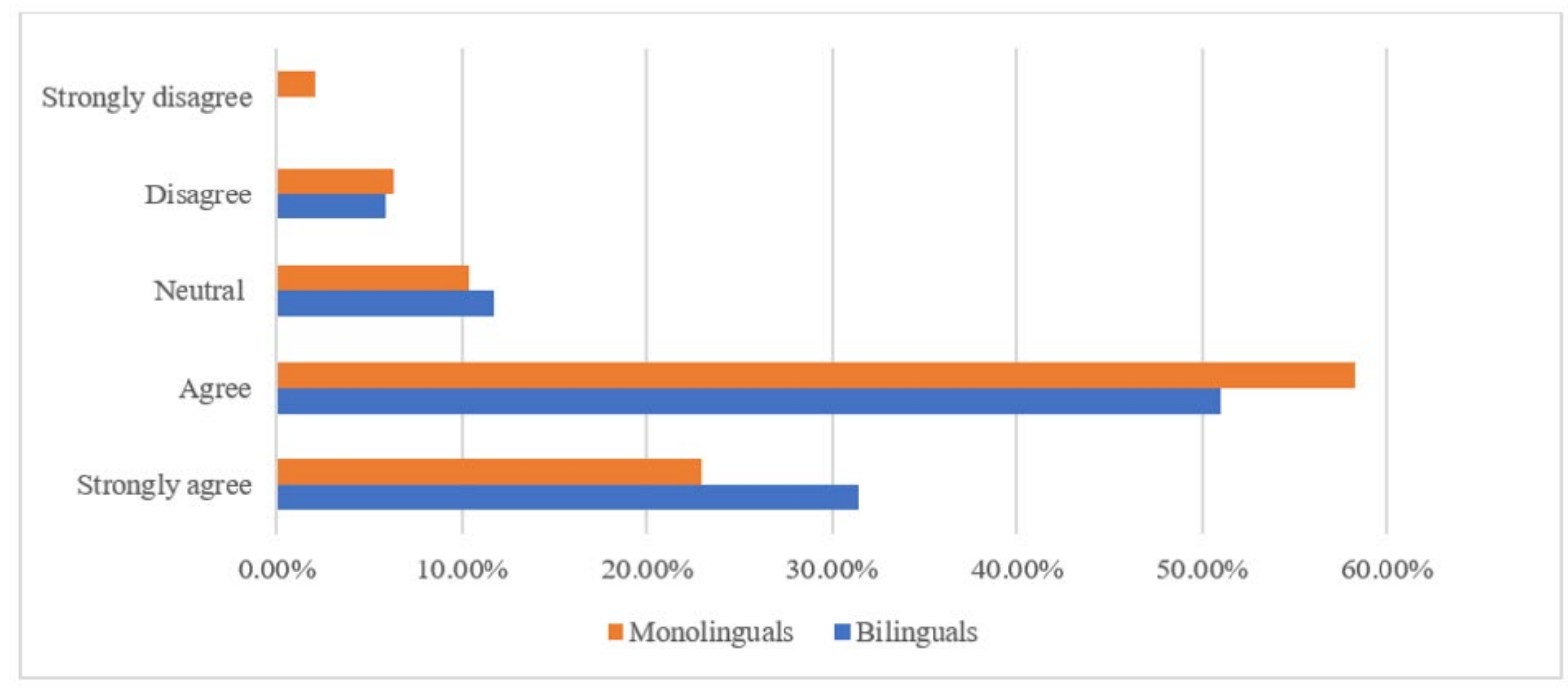

Figure 4. Range of student agreement that language benefits cognition

The statement that bilingual students have a cognitive advantage over monolinguals was agreed upon by bilinguals $(52.90 \%)$ but not corroborated by monolinguals $(37.50 \%)$. However, monolinguals did not refute this statement $(23.00 \%)$, showing no conclusive beliefs.

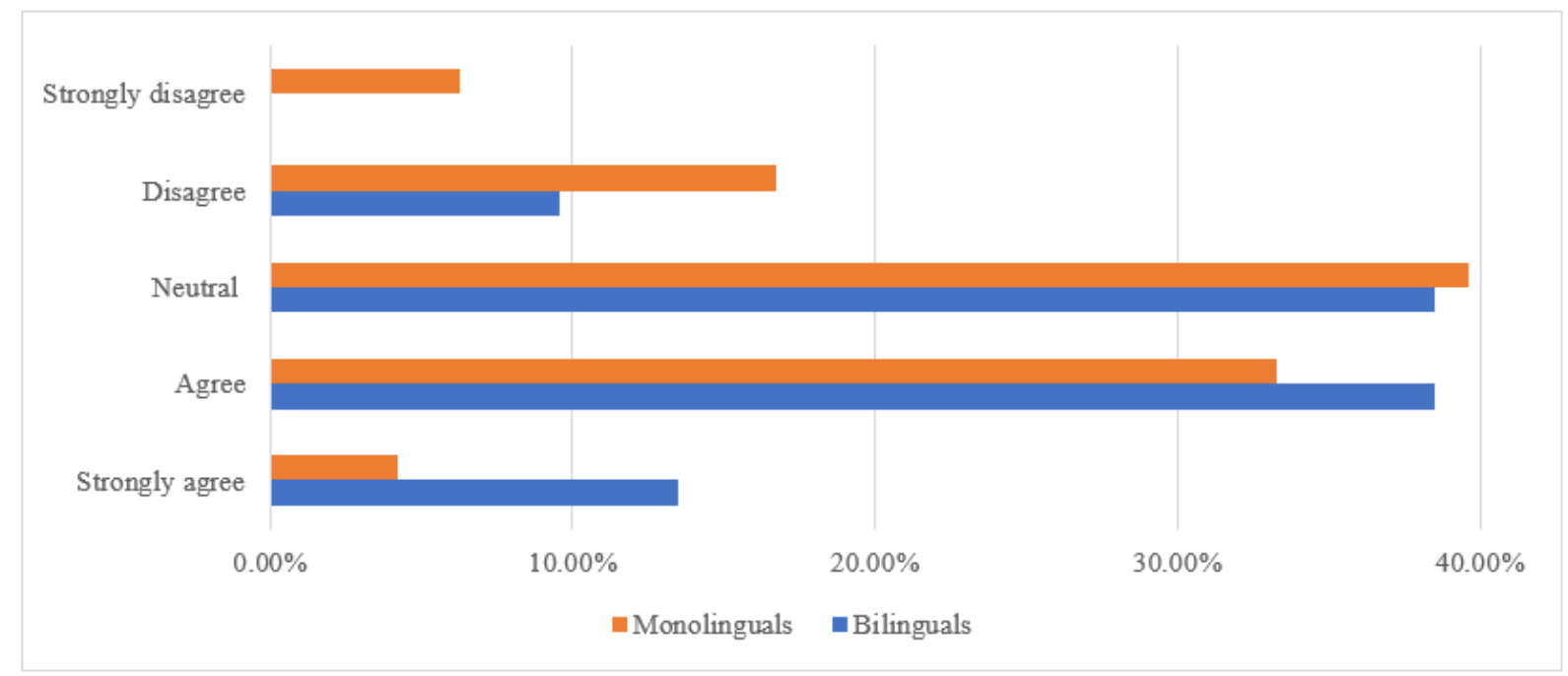

Figure 5. Range of student agreement that bilingual students have a cognitive advantage 
Neither bilinguals (25.50\%) nor monolinguals (8.00\%) agreed that bilingual students perform better in school. However, neither bilinguals $(17.30 \%)$ nor monolinguals $(25.10 \%)$ refuted the statement, showing no conclusive perceptions of bilingual performance in school. Additionally, a clear majority for neutral responses was observed for both bilinguals $(57.70 \%)$ and monolinguals $(66.70 \%)$.

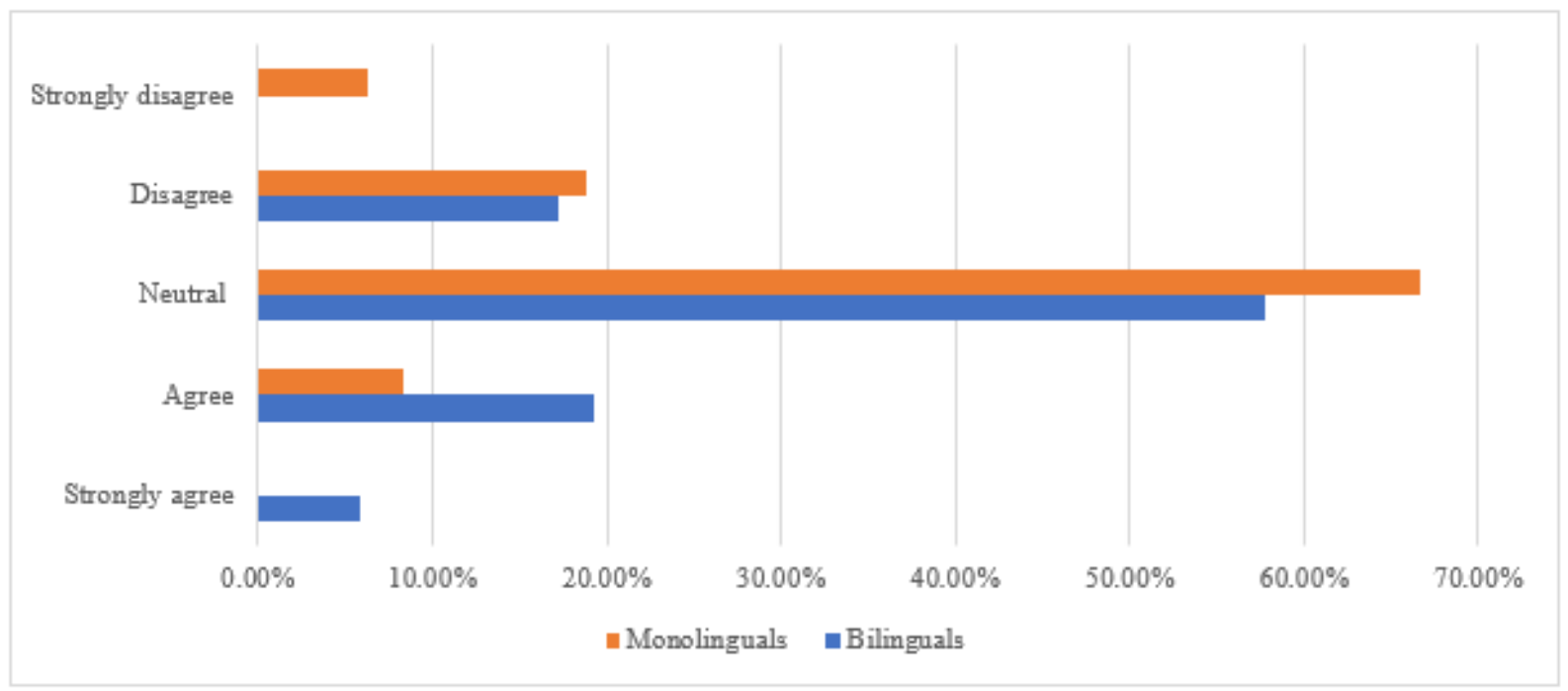

Figure 6. Range of student agreement that bilingual students perform better in school

Monolinguals believed that learning another language would benefit them cognitively $(85.40 \%)$. However, for the remaining two statements, monolinguals neither agreed nor disagreed while also not favoring neutrality.

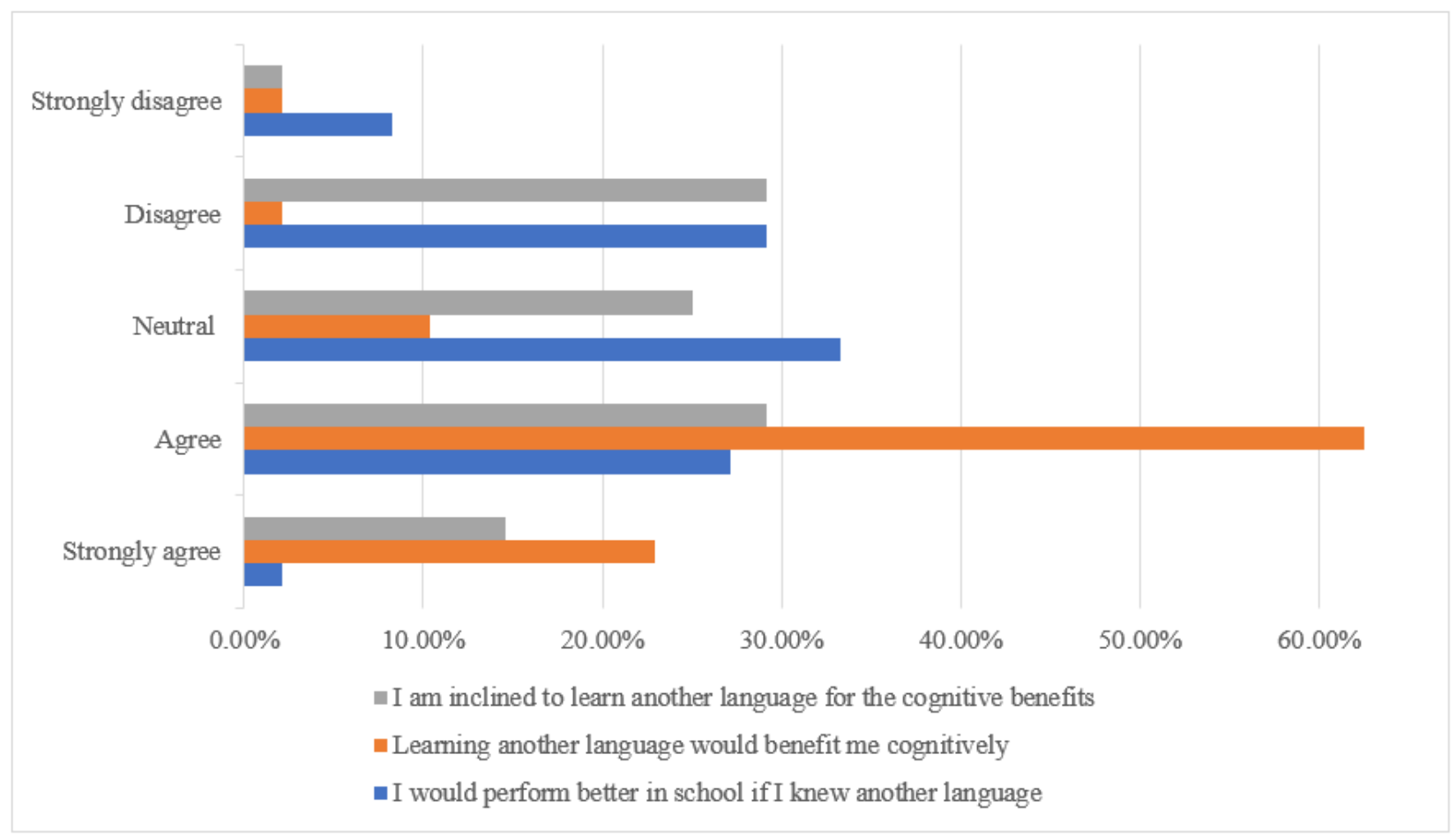

Figure 7. Range of monolinguals' agreement on statements regarding learning another language 


\section{Self-Assessments}

After completing the survey, 23 students took the cognitive assessments, 9 bilinguals and 14 monolinguals. The mean score of bilinguals on the flanker task $(\mathrm{M}=74.56)$ was higher than the mean score of monolinguals $(\mathrm{M}=$ 66.57). The difference between the scores of the language groups is statistically significant $(p=0.03)$. While the mean score on the modified flanker task of bilinguals $(M=67.67)$ was also higher than the mean score of monolinguals $(M=63.14)$, the difference is not statistically significant $(\mathrm{p}=0.17)$.

Table 1. Mean cognitive scores (/75) and SD of bilinguals and monolinguals on the flanker and modified flanker tasks

\begin{tabular}{lllllll}
\hline & \multicolumn{5}{l}{ Flanker } & \multicolumn{2}{c}{ Modified Flanker } & \\
\cline { 2 - 5 } & $\mathrm{M}$ & $\mathrm{SD}$ & $\mathrm{M}$ & $\mathrm{SD}$ & $\mathrm{P}(<0.05)$ & $\mathrm{N}$ \\
\hline Bilingual & 74.56 & 0.73 & 67.67 & 8.65 & 0.02 & 9 \\
Monolingual & 66.57 & 13.95 & 63.14 & 11.94 & 0.54 & 14 \\
$\mathrm{P}(<0.05)$ & 0.03 & & 0.17 & & & \\
\hline
\end{tabular}

Large standard deviation (SD) was observed in the data set of monolinguals in the flanker (13.95) and modified flanker (11.94) task as well as the bilingual flanker task (8.65). Due to this, the median was calculated to better account for the outliers than the mean. This, however, does not alter the previously derived statistical significance as the p-test investigates a null hypothesis, not reliant on the mean nor median of the data set.

Table 2. Mean, median (MED) and \% accuracy of bilingual and monolingual cognitive scores (/75) on the flanker and modified flanker tasks

\begin{tabular}{lccccccc}
\hline & \multicolumn{1}{l}{ Flanker } & \multicolumn{5}{c}{ Modified Flanker } \\
\cline { 2 - 6 } & M & MED & \%MED & M & MED & \%MED & N \\
\hline Bilingual & 74.56 & 75 & 100.00 & 67.67 & 71.00 & 94.67 & 9 \\
Monolingual & 66.57 & 73.50 & 98.00 & 63.14 & 69.00 & 92.00 & 14 \\
\hline
\end{tabular}

The $\%$ accuracy of the median scores were calculated to compare to self-assessment rankings. The median scores were used instead of the mean to better model the data with outliers. Percentages were used as proxies, correlated to GPAs based on the American grading system and to corresponding self-assessment rankings of well below to well above average. On both tasks, the language groups garnered a well above average categorization, except the monolingual group on the modified flanker task, earning an above average. 
Table 3. Corresponding median \% accuracy scores and GPAs to self-assessment rankings

\begin{tabular}{|l|l|l|}
\hline Ranking & Accuracy (\%) & GPA \\
\hline Well above average & $93-100$ & 4.0 \\
\hline Above average & $87-92$ & $3.3-3.7$ \\
\hline Average & $83-86$ & 3.0 \\
\hline Below average & $73-82$ & $2.0-2.7$ \\
\hline Well below average & $<73$ & $<2.0$ \\
\hline
\end{tabular}

Table 4. Self-assessment categorization associated with the median \% accuracy on the flanker and modified flanker tasks

\begin{tabular}{lll}
\hline & Flanker & Modified Flanker \\
\hline Bilingual & Well above average & Well above average \\
Monolingual & Well above average & Above average \\
\hline
\end{tabular}

The most selected categorization of academic performance for monolinguals was above average (64.60\%) and well above average (40.40\%) for bilinguals. The difference in the collapsed categorization of average, above average or well above average differed insignificantly with more monolinguals $(2.00 \%)$ rating themselves as such. There was, however, significant differences in the distribution of the self-assessments within those rankings. More bilinguals $(21.6 \%)$ rated their performance to be at the highest level. When inspecting only the above average and well above average categorizations, there was an insignificant difference $(4.5 \%)$ in favor of monolinguals.

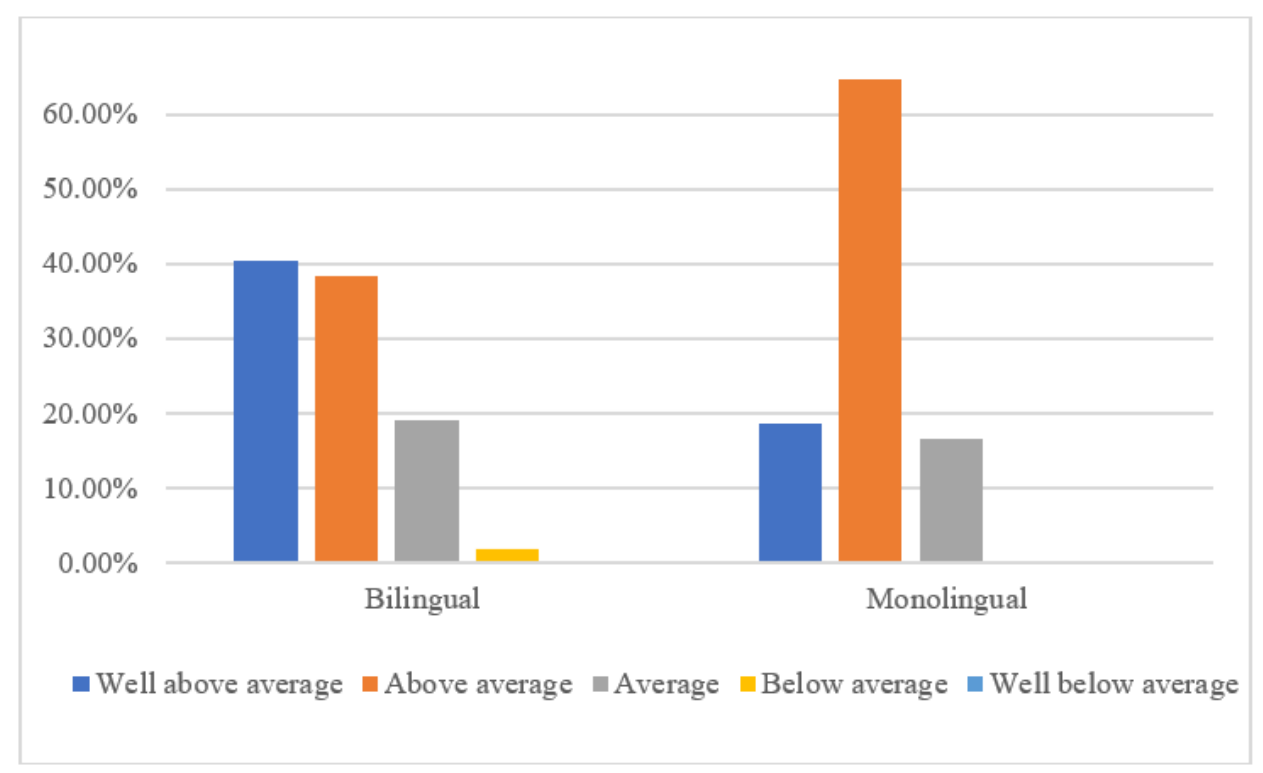

Figure 8. Self-assessment of overall academic performance 


\section{Task Demand}

Both bilinguals' and monolinguals' mean and median scores were lower on the modified flanker task than the standard flanker task. However, the difference in scores between the tasks is statistically significant for bilinguals $(p=0.02)$ but insignificant for monolinguals $(p=0.54)$, as indicated by the top $P$ column of table 1 . This indicates that bilingual performance differed between the two tasks, while monolingual scores were constant.

Students refrained from a response when a X was present among the arrows in the modified flanker task. Since the $\mathrm{X}$ count was randomized for each trial, the relation between the amount of Xs per trial and correct responses was examined to further observe task demand.

Bilingual cognitive scores worsened with an increased $X$ count $(m=-0.70)$, however the negative linear correlation presented a weak fit for the data $\left(R^{2}=0.08\right)$. The trend for monolingual responses, opposite bilingual performance, improved with an increased $X$ count $(m=0.57)$. Similarly, to the bilingual regression line, the monolingual trendline poorly modeled the data $\left(R^{2}=0.02\right)$.

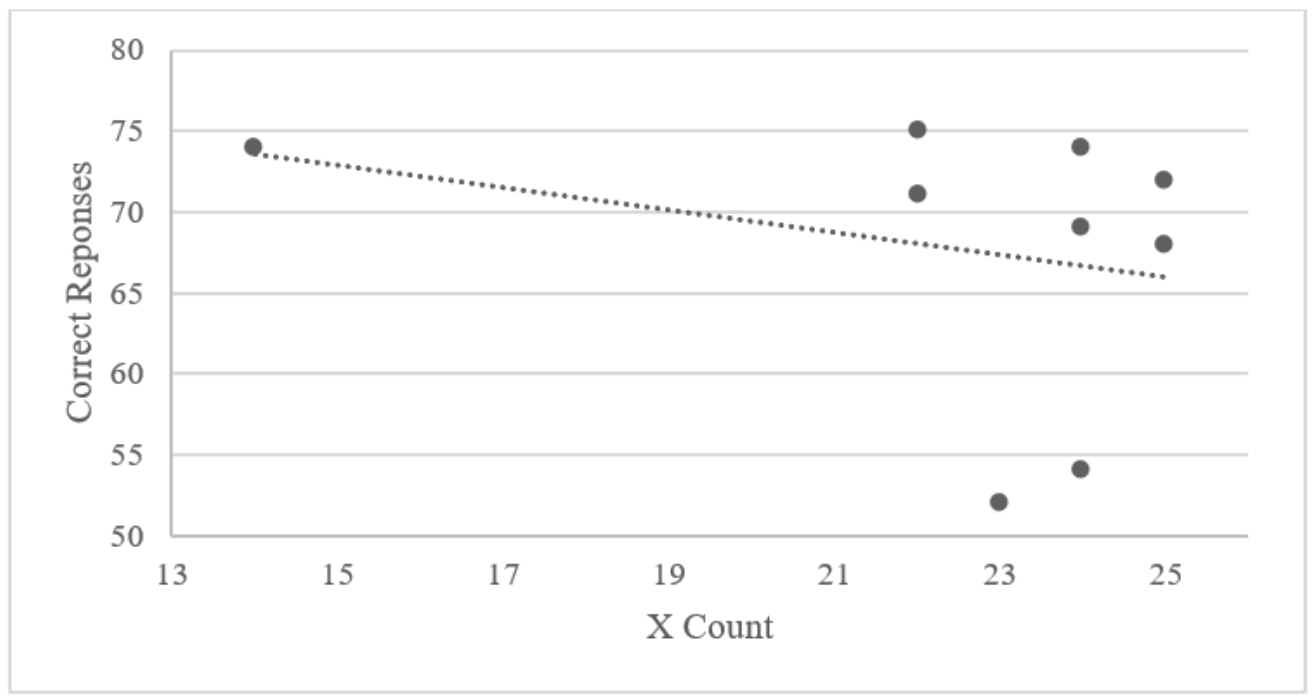

Figure 9. Correlation of correct bilingual responses to $\mathrm{X}$ count in the modified flanker task

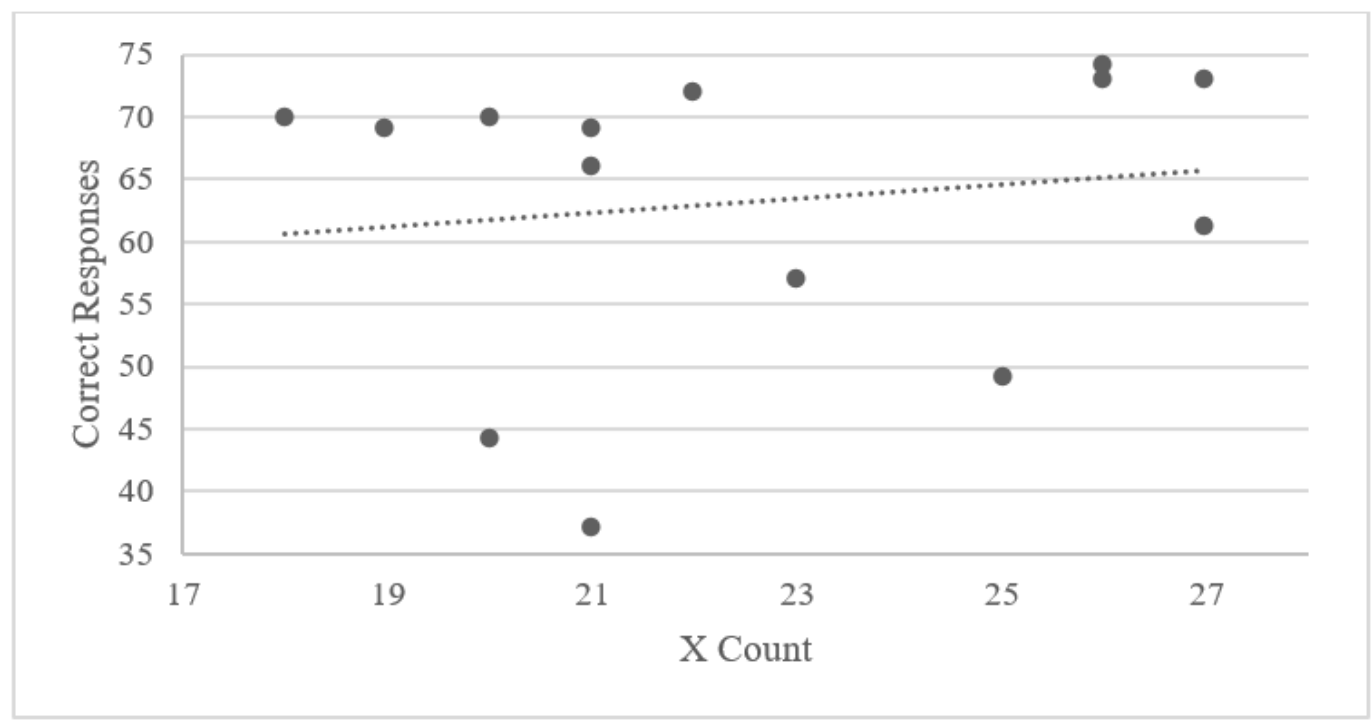

Figure 10. Correlation of correct monolingual responses to $X$ count in the modified flanker task 


\section{Analysis}

\section{Student Perception}

Both language groups accepted that language benefits cognition. This demonstrates that student perception of language aligns with the BA hypothesis. This alignment further highlights the long-held belief of superior bilingual cognition common in seminal research. Despite such results becoming increasingly disproved in favor of the null hypothesis theory due to methodological inconsistencies, as in Paap et al.'s (2015) and Adescope et al. (2010) meta- analysis, the perception of bilingualism remains constant.

Only bilinguals believed that bilingual students have a cognitive advantage and neither group reported that bilingual students perform better in school. This indicates that students do not perceive the direct linear relationship between cognition and school trajectories that was discovered by Guhn et al. (2016) by investigating student classification based on the EDI and Hair et al. (2006) who investigated predictors of school readiness. This disagreement with the cognition-school relationship is further exhibited in monolingual responses regarding learning another language as monolinguals believe that another language would benefit them cognitively but would not improve their school performance.

It is important to consider that while some statements were not agreed upon, they were not refuted either, not receiving a majority for the disagree categorization. This pertains to both statements regarding bilingual students possessing a cognitive advantage and their subsequent better performance in school. The clear majority for neutral responses regarding improved bilingual performance in school demonstrates no perception from either language group on the subject. Contrastingly, no majority for any response among monolinguals, including neutrality, occurred for the statement regarding bilingual students possessing a cognitive advantage. Even though the statements were not refuted, this nonetheless indicates disagreement with Guhn et al.'s (2016) and Hair et al.'s (2006) findings as such categorization does not favor a direct relationship, rather no relationship, between cognition and school performance.

\section{Self-Assessments}

A comparison of the student self-assessed overall academic performance and cognitive scores with their corresponding categorization revealed that bilingual self-assessments were more accurate. On both tasks, bilinguals scored well above average, consistent with their most selected self-assessment ranking. Monolinguals ranked well above average and above average on the tasks. Due to the differing categorizations, the mean of the accuracy was taken, revealing that on the tasks overall, monolinguals garnered an above average ranking (95.00\%). This demonstrates that monolinguals under-estimated their academic performance, as only $18.80 \%$ of monolinguals accurately assessed their performance. Furthermore, amongst bilinguals, the well above average ranking received a slight majority categorization as the difference between well above and above average categorization was a mere $1.9 \%$. This demonstrates that while the bilingual rating was more accurate, monolinguals' selfassessments were more consistent among the language group as a whole. Still, the close to evenly split well above and above average categorization among bilinguals demonstrates that their self-assessments also underestimate their academic performance, however, to a lesser extent than those of monolinguals.

\section{Task Demand}

The modified flanker task increased WM function and thus task demand by introducing a new constraint for responses, as demonstrated by Lu et al. (2019). Since both mean and median scores were lower on the modified flanker task, this demonstrates consistent inferior performance irrespective of outliers, represented by the large 
SD. The statistical significance among the data sets demonstrates that bilingual performance worsened on the modified flanker task compared to the standard flanker task while monolingual performance remained constant. This shows that bilingual performance was negatively impacted by increased WM task demand while monolingual scores were unimpacted. Such findings refute the ECT as no scores improved when executive task demand was increased, predicting more efficient resource allocation, as explained by Antón et al. (2019). This contradicts the findings of both Antón et al. (2019) and Lu et al. (2019) that discovered improved cognitive performance with increased WM task demand, codified in the ECT, thus, supporting it. These studies only observed a BA in the highest demand trials: backward Corsi and digit span WM tasks in Antón et al.'s study and the final trial of the Conditional-GNG in Lu et al.'s work. Furthermore, Lu et al.'s findings are opposite the current research as bilinguals performed better on the higher WM demand tasks, the modified flanker, but scored equally on the standard flanker task. Current findings support the first hypothesis presented by Lu et al., stating worsened performance with increased cognitive load while Lu et al. corroborated the second hypothesis.

An aspect of Antón et al.'s and Lu et al.'s findings that are supported by current results is the presence of a selective BA, regardless of the task demand associated with it. A statistical significance was only observed between monolingual and bilingual scores on the standard flanker task, in favor of bilinguals. This shows an inconsistent BA only present on lower demand task, similar to the findings of Antón et al. and Lu et al., observing a nonconstant BA, only occurring under certain conditions. The necessary conditions for the presence of a BA differed, however, from current findings, as previously discussed. This demonstrates that the BA is dependent on multiple variables, not guaranteed solely by bilingualism. Moreover, this shows that bilinguals outperform their monolingual peers on school tasks employing low demand WM usage, while equally on activities with high demand.

In addition to the new constraints introduced in the modified flanker task, the amount of Xs per trial impacted task demand as a higher $\mathrm{X}$ count increased EF. The low correlation coefficient $\left(R R^{2}\right)$ of the trendlines that modeled the relationship between the $\mathrm{X}$ count and cognitive scores demonstrates the amount of Xs present per task did not impact performance for either language group. This also disproves the ECT as an increased X count and thus increased task demand did not positively impact performance.

These findings differ from those of Lu et al. (2019), who also used the standard flanker to test low WM load while the modified flanker examined higher demands. Lu et al. (2019) introduced conditions to further vary task demand by adding target, distractor, and lure trials to the Conditional-GNG task. This is comparable to the fluctuating $\mathrm{X}$ count in modified flanker trials as new stimuli was added in both conditions to vary task demand. Lu et al. (2019) discovered a BA in lure trials, which required the highest WM capacity. Contrastingly, no impact on performance was associated with $\mathrm{X}$ count in the current findings.

\section{Conclusion}

Using cognitive scores as proxies for academic trajectories, the data shows that bilingualism positively impacts academic performance on tasks with lower WM demand. For academic tasks that require greater WM, bilingual academic performance worsens, but becomes equal to that of monolinguals. Therefore, a BA is selectively and dependently present in school performance, impacted by the cognitive task type and load necessary to complete the curriculum.

While proxy categorization of cognitive scores was an appropriate measure of school performance, as demonstrated by the relationship between cognition and education discovered by Guhn et al. (2016) and Hair et al. (2006), direct categorization based on GPAs would provide the most accurate academic success trajectory model. Therefore, further research is necessary to examine GPAs of bilingual and monolingual students to compare overall academic performance between language groups. In this study, due to privacy constraints, the flanker and modified flanker tasks were used to gather information on students' academic abilities instead of GPAs. Additionally, the sample population of the cognitive assessments was not ideal for the data collection, introducing possible QRPs. As demonstrated by Paap et al. (2015), a smaller sample size is more susceptible to false positives. Furthermore, 
the difference in sample size between bilinguals and monolinguals on the flanker tasks created outliers that bore significant impacts on the means of the data. Although this was controlled to some extent by analyzing medians, taking outliers into account, this nonetheless introduced variance. Therefore, a future study with a larger, more homogeneous interlanguage sample size is necessary to verify current findings.

To further eliminate QRPs, the current study should be replicated with increased controls to confirm that the differences in cognitive scores were derived solely from bilingualism. This is especially important to examine when considering that a selective BA was discovered among the cognitive assessments, showing non-constant results that may have resulted from a chance factor. Although Bialystok and Calvo (2014) and Adescope et al. (2010) justify decreased controls, replicating current results with minimized uncontrolled variables would eliminate the possibility of outside influences on results.

Finally, to confirm the valid application of BA findings in WM to all aspects of cognition, other sectors of EF must be tested through cognitive assessments between language groups. This would determine if current findings on the BA, its selective properties, and the relation to task demand are specific to WM rather than cognition as a whole.

This study offers several contributions to the scholarly discussion of the BA, especially considering the current contradictions to seminal works. First, the findings disprove the co- dependence of the BA and ECT, as the BA was not present among the modified flanker task, despite increased demands on WM. Next, it has been established that the BA is an inconsistent occurrence, not automatically present solely due to one's bilingual abilities. Rather, the BA is dependent on the type of cognitive processes that are activated and their load. In this study, no consistent BA was discovered. Rather, a singular instance of the BA was observed on the low WM demand tasks, opposite to previous findings that established either a null or positive relationship between BA and task demand. This data shows a negative relationship between cognitive performance and task demand among bilinguals, favoring the first hypothesis of cognitive load.

Lastly, these findings can be applied to the classroom to maximize instruction for students with varying linguistic abilities. By recognizing the inferior performance of bilingual students on tasks with higher WM demands, such skills can be emphasized for those students. This can especially be promoted in ELL programs, encompassing $10.1 \%$ of America students according to the National Center for Education Statistics (English language learners, 2019). The degree of proficiency varies in ELL programs, similarly to this study as participants were not grouped by fluency in their languages. Therefore, these findings can be applied to an emerging bilingual ELL population.

The perception survey revealed that students do not recognize the link between cognition and school performance. Therefore, if students, especially monolinguals, were made aware of the scholarly discussion surrounding the BA, this could encourage more high schoolers to study another language. This is supported by the monolingual survey that favored learning another language but did not recognize the associated cognitive or school benefits with such an endeavor. If monolinguals are motivated to learn another language, this could bridge the cognitive gap between the differing language groups and create a more equal academic experience for all students.

\section{Acknowledgments}

The author would like to thank Jill Berge for guiding the creation of this paper and providing valuable feedback on earlier drafts. The author is grateful to all the participants in this study who contributed their time to further bilingual research. 


\section{References}

Adescope, O. O., Lavin, T., Thompson, T., \& Ungerleider, C. (2010). A Systematic Review and Meta-Analysis of the Cognitive Correlates of Bilingualism. Review of Educational Research, 80(2), 207-245. https://doi.org/10.3102/0034654310368803

America speaks: a demographic profile of foreign-language speakers for the United States: 2000. (2000). Census 2000. United States Census Bureau.

Antón, Eneko, Carreiras, Manuel, \& Duñabeitia, Jon Andoni. (2019). The impact of bilingualism on executive functions and working memory in young adults. PloS One, 14(2), E0206770.

Calvo, A., \& Bialystok, E. (2014). Independent effects of bilingualism and socioeconomic status on language ability and executive functioning. Cognition, 130(3), 278-288. https://doi.org/10.1016/j.cognition.2013.11.015

Children who speak a language other than English at home in the United States. (2019). Kids Count Data Center.

Engel de Abreu, P. M. J., Cruz-Santos, A., Tourinho, C. J., Martin, R., \& Bialystok, E. (2012). Bilingualism Enriches the Poor: Enhanced Cognitive Control in Low-Income Minority Children. Psychological Science (0956-7976), 23(11), 1364-1371. https://doi.org/10.1177/0956797612443836

English language learners in public schools. (2019). Condition of Education, 1-4. National Center for Education Statistics.

Eriksen, Barbara A, \& Eriksen, Charles W. (1974). Effects of noise letters upon the identification of a target letter in a nonsearch task. Perception \& Psychophysics, 16(1), 143-149.

Felt, C. (2017). King County's Changing Demographics: Investigating our Increasing Diversity. Regional Law, Safety \& Justice Committee.

Guhn, M., Milbrath, C., \& Hertzman, C. (2016). Associations between child home language, gender, bilingualism and school readiness: A population-based study. Early Childhood Research Quarterly, 35, 95-110. https://doi.org/10.1016/j.ecresq.2015.11.003

Gutiérrez-Clellen, V. F., Calderén, J., \& Weismer, S. E. (2004). Verbal Working Memory in Bilingual Children. Journal of Speech, Language \& Hearing Research, 47(4), 863-876. https://doi.org/10.1044/1092-4388(2004/064)

Hair, E., Halle, T., Terry-Humen, E., Lavelle, B., \& Calkins, J. (2006). Children's school readiness in the ECLS-K: Predictions to academic, health, and social outcomes in first grade. Early Childhood Research Quarterly, 21(4), 431-454. https://doi.org/10.1016/j.ecresq.2006.09.005

Han, W.-J. (2012). Bilingualism and academic achievement. Child Development, 83(1), 300-321. https://doi.org/10.1111/j.1467-8624.2011.01686.x

International Migration Report. (2019). Department of Economic and Social Affairs, Population Division. United Nations.

Lu Jiao, Baoguo Chen, Cong Liu, \& Ruiming Wang. (2019). Working memory demand of a task modulates bilingual advantage in executive functions. International Journal of Bilingualism, 23(1), 102-117. https://doi.org/10.1177/1367006917709097

Paap, K. R., Johnson, H. A., \& Sawi, O. (2015). Bilingual advantages in executive functioning either do not exist or are restricted to very specific and undetermined circumstances.

Cortex, 69, 265-278. https://doi.org/10.1016/j.cortex.2015.04.014

The number of bilingual kids in America continues to rise. (2018). Kids Count Data Center. Annie E. Casey Foundation.

Waters, M. C., \& Gerstein, M. A. (2015). Data on immigrants and immigrant integration. In The integration of immigrants into American society (pp. 413-435). National Academies Press. doi.org/10.17226/21746 